\title{
Magnetic Phase Diagram of the Hubbard Model
}

\author{
J. K. Freericks ${ }^{1, *}$ and Mark Jarrell ${ }^{2}$ \\ ${ }^{1}$ Department of Physics, University of California, Davis, California 95616 \\ ${ }^{2}$ Institute for Theoretical Physics, University of California, Santa Barbara, California 93106 \\ and Department of Physics, University of Cincinnati, Cincinnati, Ohio 45221
}

(Received 28 July 1994)

\begin{abstract}
The competition between commensurate and incommensurate spin-density-wave phases in the infinite-dimensional single-band Hubbard model is examined with quantum Monte Carlo simulation and strong and weak coupling approximations. Quantum fluctuations modify the weak-coupling phase diagram by factors of order unity and produce remarkable agreement with the quantum Monte Carlo data, but strong-coupling theories (that map onto effective Falicov-Kimball models) display pathological behavior. The single-band model can be used to describe much of the experimental data in $\mathrm{Cr}$ and its dilute alloys with $\mathrm{V}$ and $\mathrm{Mn}$.
\end{abstract}

PACS numbers: 75.30.Kz, 71.27.+a, 75.10.Lp

Spin-density-wave (SDW) order, in which the modulation wave vector of the SDW is incommensurate with the underlying lattice, is one of the most fascinating ordered states found in nature. Incommensurate magnetism occurs in both metallic and insulating phases and on both frustrated and unfrustrated lattices. In general, incommensurate magnetic order may be driven either by frustration or by Fermi surface nesting with a wave vector that lies away from commensurate wave vectors. It is important to understand which process plays a more important role and to understand how many-body effects modify the stability of incommensurate phases. Here the effect of nesting is examined on an unfrustrated lattice with strongly correlated electrons. The resulting phase diagram is then compared to approximate results in the weak- and strong-coupling limits (Figs. 1 and 2). Finally, our theoretical results are compared to those found in $\mathrm{Cr}$ and its dilute alloys.

Elemental $\mathrm{Cr}$ is a paradigm for an antiferromagnetic metal [1] with incommensurate SDW order driven by Fermi-surface nesting. The lattice structure of $\mathrm{Cr}$ is an unfrustrated body-centered-cubic structure which may be modeled by a Hubbard model [2] near half filling with moderate electron-electron correlations. Adding electrons to $\mathrm{Cr}$ (by alloying with $\mathrm{Mn}$ ) rapidly makes the magnetic order commensurate with the lattice, whereas removing electrons from the system (by alloying with $\mathrm{V}$ ) rapidly increases the incommensuration and decreases the magnetic transition temperature, eventually to zero [3].

Heretofore, incommensurate magnetic order has mainly been examined within the Hartree-Fock (HF) (weak-coupling) approximation that neglects quantum fluctuations. Penn [4] found incommensurate order in the three-dimensional Hubbard model, and Schulz [5] found evidence for incommensurate phases on a square lattice.

In this contribution the magnetic phase diagram of the single-band Hubbard model is investigated in the limit of infinite dimensions [6]. This limit is useful, because it has been shown to contain most of the physics expected of three-dimensional Hubbard models, and the many-body effects can be treated numerically with the quantum Monte Carlo (QMC) techniques of Hirsch and Fye [7]. This allows us to demonstrate the existence of incommensurate order at finite temperatures in a model that only includes Fermi-surface nesting effects and electron-electron correlations.

The Hubbard model [2] is described by the following Hamiltonian:

$$
\begin{aligned}
H= & -\frac{t^{*}}{2 \sqrt{d}} \sum_{\langle i j\rangle \sigma}\left[c_{i \sigma}^{\dagger} c_{j \sigma}+c_{j \sigma}^{\dagger} c_{i \sigma}\right] \\
& +U \sum_{i}\left(n_{i \uparrow}-\frac{1}{2}\right)\left(n_{i \downarrow}-\frac{1}{2}\right)-\mu \sum_{i \sigma} n_{i \sigma},
\end{aligned}
$$

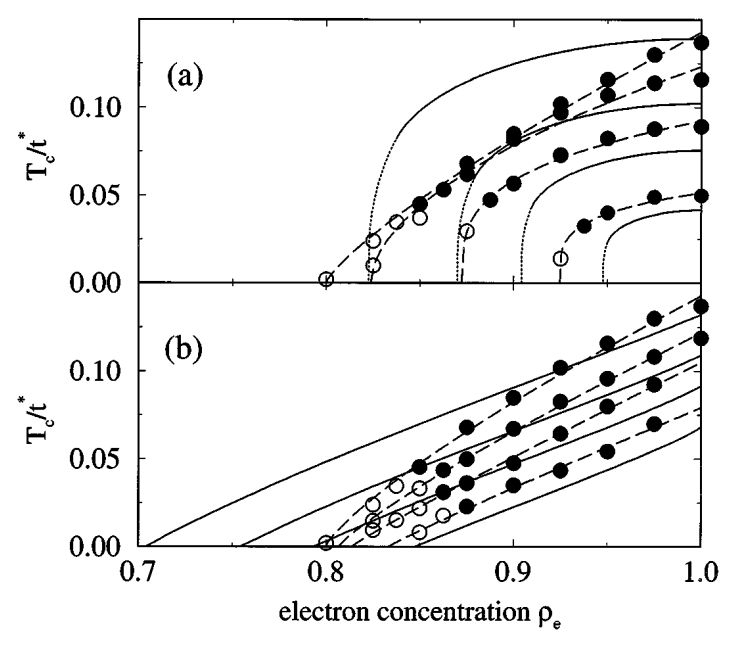

FIG. 1. Phase diagram of the Hubbard model in the (a) weakcoupling regime $\left(U / t^{*}=1,1.5,2,3\right)$ and (b) strong-coupling regime $\left(U / t^{*}=3,4,5,7\right)$. The solid (open) dots denote the transition temperature to a commensurate (incommensurate) SDW phase as determined by a QMC calculation. The solid (dotted) lines denote the transition temperature to a commensurate (incommensurate) SDW phase using the modified Stoner criterion in (a) and using Li and d'Ambrumenil's approximation in (b). The dashed lines are a fit of the QMC data by the form $T_{c} / t^{*}=a\left(\rho-\rho_{c}\right)^{c}$. The exponent $c$ increases with increasing $U$. 


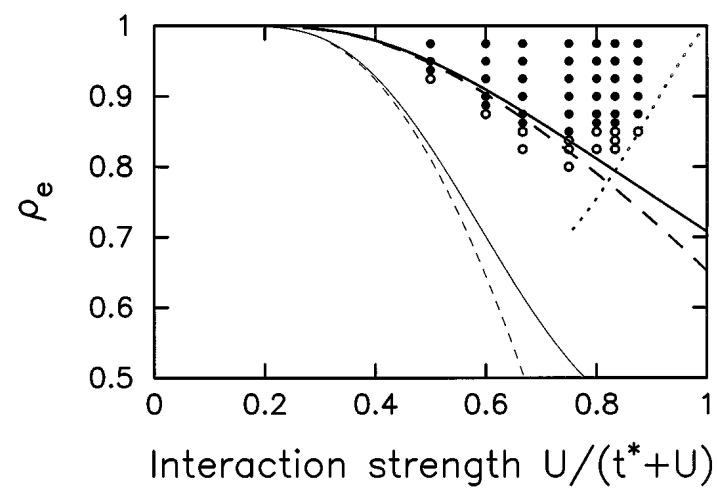

FIG. 2. Phase diagram for the Hubbard model as a function of electron concentration and $U$. The thin (thick) solid lines denote the commensurate-incommensurate phase boundary for the Stoner criterion (modified Stoner criterion); the thin (thick) dashed lines are the corresponding results for the incommensurate-paramagnetic phase boundary. The dotted line is the strong-coupling approximation for the commensurateparamagnetic phase boundary. The solid (open) dots denote the QMC solutions that display commensurate (incommensurate) SDW order.

where $c_{i}^{\dagger}\left(c_{i}\right)$ is a creation (destruction) operator for an electron at site $i$ with spin $\sigma$. The hopping matrix elements connect nearest neighbors on a hypercubic lattice in $d$ dimensions; its magnitude is written as $t=t^{*} / 2 \sqrt{d}$ [to have a well-defined limit in infinite dimensions $(d \rightarrow \infty)]$. All energies are expressed in units of the rescaled hopping matrix element $t^{*}$. The Coulomb repulsion is represented by $U$ and the chemical potential by $\mu$.

In the limit of infinite dimensions the local approximation becomes exact [6]. The electronic Green's function $G\left(i \omega_{n}\right) \equiv G_{n}$ is represented by an integral over the noninteracting density of states $\rho(y)=\exp \left(-y^{2}\right) / \sqrt{\pi}$ :

$$
\begin{aligned}
G_{n} & =\int_{-\infty}^{\infty} d y \frac{\rho(y)}{i \omega_{n}-\mu-\Sigma_{n}-y} \\
& \equiv F_{\infty}\left(i \omega_{n}+\mu-\Sigma_{n}\right),
\end{aligned}
$$

with $\Sigma_{n} \equiv \Sigma\left(i \omega_{n}\right)$ the electronic self-energy. The magnetic susceptibility satisfies Dyson's equation,

$$
\chi_{m n}(\mathbf{q})=\chi_{m}^{0}(\mathbf{q}) \delta_{m n}-T \sum_{r} \chi_{m}^{0}(\mathbf{q}) \Gamma_{m r} \chi_{r n}(\mathbf{q}),
$$

with $\Gamma_{m r} \equiv \Gamma\left(i \omega_{m}, i \omega_{r}\right)$ the local irreducible vertex function for SDW order. The bare particle-hole susceptibility $\chi_{m}^{0}(\mathbf{q})$ is defined by

$$
\begin{aligned}
\chi_{m}^{0}(\mathbf{q}) \equiv & -T \sum_{k} G_{m}(\mathbf{k}) G_{m}(\mathbf{k}+\mathbf{q}) \\
= & -\frac{1}{\sqrt{\pi}} \frac{1}{\sqrt{1-X^{2}}} \int_{-\infty}^{\infty} d y \\
& \times \frac{e^{-y^{2}}}{i \omega_{n}-\mu-\Sigma_{n}-y} F_{\infty} \\
& \times\left[\frac{i \omega_{n}+\mu-\Sigma_{n}-X y}{\sqrt{1-X^{2}}}\right] .
\end{aligned}
$$

The bare susceptibility only depends on the scalar param- eter $X(\mathbf{q}) \equiv \sum_{i=1}^{d} \cos \mathbf{q}_{i} / d$, which defines an equivalence class of wave vectors in the infinite-dimensional Brillouin zone [8]. $\quad X(\mathbf{q})$ can be parametrized by the line that extends along the Brillouin zone diagonal from the zone center $(X=1)$ to the zone corner $(X=-1)$. The self-energy and irreducible vertex function are extracted from the self-consistent QMC simulations as described previously [9].

It is important to qualify the region of validity of different approximation techniques by comparing them with the numerically exact QMC results (the errors of the QMC calculation are well understood and controllable).

In the weak-coupling limit a renormalized Hartree-Fock approach [10] is employed. The Néel temperature is determined by the Stoner criterion,

$$
\frac{1}{U}=\chi_{p h}^{0}(X, T) \equiv T \sum_{n} \chi_{n}^{0}(X),
$$

where the bare particle-hole susceptibility $\chi_{n}^{0}(X)$ is calculated with noninteracting Green's functions $G_{n}^{0}\left[\Sigma_{n}=0\right.$ in Eq. (2)]. This HF transition temperature is reduced by factors of order 3 due to quantum fluctuations [10], even in the limit $U / t^{*} \rightarrow 0$. Quantum fluctuations modify the Stoner criterion (by subtracting the local particle-particle susceptibility) to [11]

$$
\begin{aligned}
\frac{1}{U} & =\chi_{p h}^{0}(X, T)-\chi_{p p}^{0}(X=0, T) \\
& =\chi^{0}(X, T)-T \sum_{n}\left|G_{n}^{0}\right|^{2},
\end{aligned}
$$

in the limit $U / t^{*} \rightarrow 0$. These fluctuations initially reduce $T_{c}$ by the factor $\exp \left[-\chi_{p p}^{0}\left(0, T_{c}\right) / \rho(\mu)\right]$ in the weakcoupling limit $[10,11]$.

In the strong-coupling limit the Hubbard model can be mapped onto a Falicov-Kimball model $[12,13]$. This mapping is exact for the self-energy, but not for the irreducible vertex functions in the limit $U / t^{*} \rightarrow \infty$. As a result, the strong-coupling theories display pathological behaviors. More explicitly, these approximations assume that the down-spin particles form a static background when the up-spin particles move and vice versa; this system is then described by Falicov-Kimball models [14] for both the spin-up and spin-down electrons that are selfconsistently coupled together. Two different coupling schemes have been proposed so far [12,13]. Janiš and Vollhardt's approximation [12] underestimates the SDW susceptibility at half filling which strongly suppresses $T_{c}$ and does not reproduce the Heisenberg limit of $T_{c} \approx$ $t^{* 2} / 2 U$. Li and d'Ambrumenil's approximation [13] is correct for large $U$ at half filling, but has the pathological behavior of predicting ferromagnetism away from half filling because of segregation in the effective FalicovKimball model. This latter pathology occurs because the zero temperature occupation number of the static particles is $0,0.5$, or 1 , and segregation occurs whenever the static particle concentration is 0.5 and does not equal the mobile particle concentration [15]. We deal with this pathology, by only considering ordered states with $X<0$. 


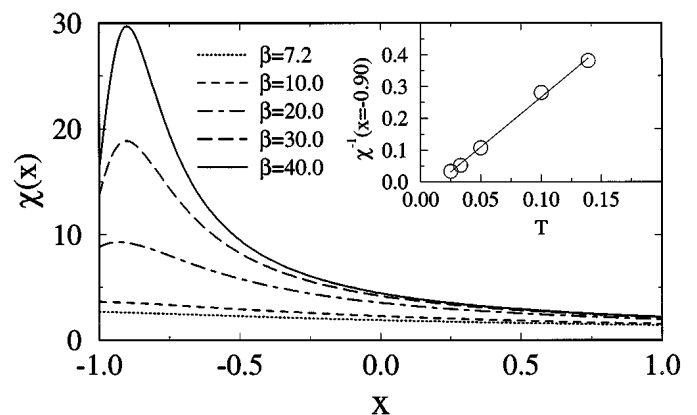

FIG. 3. The magnetic susceptibility for all $X(\mathbf{q})$ at various temperatures when $U=4$ and $\rho_{e}=0.825$. The susceptibility displays a peak at $X \approx-0.90$. As shown in the inset, the transition temperature $\left(T_{c}=0.0148 t^{*}\right)$ was inferred from extrapolation of the peak inverse susceptibility.

To determine the magnetic transition temperatures, we calculated the magnetic susceptibility for all $X(\mathbf{q})$ in the Brillouin zone. As shown in Fig. 3, the susceptibility always displayed a maximum at a distinct value of $X$. The transition temperature was then inferred from interpolation (or extrapolation) of the peak inverse susceptibility, as shown in the inset to Fig. 3. At half filling [9] the Hubbard model in infinite dimensions has a transition to a commensurate antiferromagnetic state $(X=-1)$ at the Néel temperature $T_{N}$. As the system is doped away from half filling, the Néel temperature drops until a critical filling is reached where the commensurate SDW becomes incommensurate. This is shown in Figs. 1(a) and 1(b) for the weak-coupling and strong-coupling results, respectively. As the system is doped further away from half filling, the wave vector of the ordered phase changes continuously with the electron concentration until $T_{c}$ drops to zero at the incommensurate-paramagnetic phase boundary. The shape of the magnetic phase boundary changes continuously from a square-root-like dependence (as a function of doping) at weak coupling to an almost linear dependence at strong coupling, with the same crossover region $\left(U \approx 3 t^{*}\right)$ as found for $T_{N}$ at half filling.

Phase diagrams in the weak-coupling regime $\left(U / t^{*} \leq\right.$ 3) have been obtained from both QMC simulations and from the theory of Eq. (6). They are plotted in Fig. 1(a) for four values of $U / t^{*}\left(U / t^{*}=1,1.5,2,3\right)$. Let $X_{\max }$ denote the largest value of the scalar parameter $X$ with which incommensurate order is found for each value of $U$. In the QMC simulations, we find that both $X_{\max }$ increases, and that the ratio of the transition temperature at the commensurate-incommensurate phase boundary $T_{I}$ to the Néel temperature at half filling $T_{N}$ decreases, as the coupling strength increases. However, when the modified Stoner criterion is used, we find that the transition temperature curves scale with the coupling strength and maintain the same approximate shape. This implies that $X_{\max }$ will increase, while the ratio $T_{I} / T_{N}$ remains constant, $T_{I} / T_{N} \approx 0.57$.
QMC results in the strong-coupling regime $\left(U / t^{*} \geq 3\right)$, along with $\mathrm{Li}$ and d'Ambrumenil's approximation for $T_{c}$ are plotted in Fig. 1(b) for $U / t^{*}=3,4,5,7$. The approximate results are generated with the restriction that only ordered states with $X<0$ are considered (which suppresses the ferromagnetism due to phase separation). Under this assumption, the strong-coupling theory predicts no incommensurate order (near $X=-1$ ), and the transition temperature curves also maintain the same shape as the coupling strength changes. Accurate simulations at very large values of $U$ are not possible with the QMC. Thus, we are unable to determine whether $X_{\max }$ continues to increase in the strong-coupling regime, nor are we able to determine what happens to $T_{I} / T_{N}$.

Both weak- and strong-coupling approximations are unable to reproduce the qualitative change in shape of the finite-temperature phase diagrams as a function of $U$.

The "phase diagram" which indicates the commensurate-incommensurate phase boundary (occurring at $T=T_{I}$ ) and the incommensurate-paramagnetic phase boundary (occurring at $T=0$ ) is presented in Fig. 2. The thin (thick) solid lines denote the commensurate-incommensurate boundary for the Stoner (modified Stoner) criterion; the thin (thick) dashed lines plot the corresponding paramagnetic phase boundary. The dotted line is the commensurate-paramagnetic phase boundary in the strong-coupling theory, and the dots are the QMC results. The quantum fluctuations strongly renormalize the HF phase boundary to produce good agreement with the QMC [the critical value of $U$ is shifted by $\left.1 / U=1 / U_{\mathrm{HF}}-\chi_{p p}^{0}\left(0, T_{c}\right)\right]$. The value of $X_{\max }=-0.7$ occurs at $U=\infty$ when the modified Stoner criterion is used, whereas $X_{\max }$ increases to the ferromagnetic point $(X=1)$ in the Stoner theory, i.e., quantum fluctuations completely suppress ferromagnetism in this approximation.

Finally, we comment on the possibility of phase separation. Recent analysis of the $U \rightarrow 0$ limit at $T=0$ has shown that phase separation between the commensurate SDW and the paramagnetic phase precludes the appearance of incommensurate order [16]. This phase separation disappears when $U$ is larger than $0.8 t^{*}$. We cannot directly rule out the possibility of phase separation modifying the results found here, but we have checked that the uniform compressibility is positive for all of the QMC results (a necessary but not sufficient condition against phase separation).

Finally, it is of interest to compare our results with what is known about magnetism in elemental Cr. Electronic band structure calculations [1] show that the $d$-electron concentration for $\mathrm{Cr}$ is 4.6/atom which is close to a half-filled band. Doping with Mn adds an electron to the $d$ bands, and doping with $\mathrm{V}$ removes an electron. The commensurate-incommensurate phase boundary lies at a doping of $0.3 \% \mathrm{Mn}$ and the paramagnetic phase boundary at a doping of $3.5 \% \mathrm{~V}[1,3]$. Since the density of states for $\mathrm{Cr}$ is peaked near the 
band edges, rather than the band center, it is difficult to map directly onto the Gaussian density of states (of the single-band model in infinite dimensions). Instead, we compare the ratio of the incommensurate ordering wave vector to the commensurate wave vector in order to estimate the magnitude of the Coulomb interaction. The smallest value for the ratio of the incommensurate wave vector to the commensurate wave vector is 0.92 for Cr [1,3], implying $X_{\max }=\cos (0.92 \pi)=-0.97$ for the single-band model. The approximate value of $U$ is then estimated to be $U \approx 1.9 t^{*}\left(U \approx 1.5 t^{*}\right)$ for the weakcoupling theory $(\mathrm{QMC})$. (We also found that the incommensurate wave vector changes very rapidly with doping near the commensurate-incommensurate phase boundary which is reminiscent of the first-order jump in the wave vector that is seen in Cr.) The ratio of $T_{I}$ $(325 \mathrm{~K})$ to $T_{N}(700 \mathrm{~K})$ is 0.46 which is smaller than the weak-coupling value of 0.57 and is consistent with the assignment of a small value to $U / t^{*}$. The Néel temperature at half filling is approximately $0.096 t^{*}\left(0.086 t^{*}\right)$ in the weak-coupling theory (QMC) which yields an effective bandwidth $W \equiv 4 t^{*}=2.4 \mathrm{eV}(2.7 \mathrm{eV})$ for the singleband model. This is a reasonable number since $\mathrm{Cr}$ has a bandwidth of $6.8 \mathrm{eV}$, and the pileup of the density of states at the band edges [1] implies that the bandwidth for an effective single-band model must be larger than the naive approximation of one-fifth of the total bandwidth.

In conclusion, we have shown that incommensurate SDW order exists in the infinite-dimensional Hubbard model as one dopes away from half filling. A simple modification (due to quantum fluctuations) of the usual Stoner criterion produces good agreement with the weakcoupling QMC results and is easy to implement in arbitrary dimensions (the q-dependent spin susceptibility is reduced by the local particle-particle susceptibility before applying the Stoner criterion). Since these quantum fluctuations produce large renormalizations of the magnetic phase boundaries, it is worthwhile to repeat previous Hartree-Fock calculations [4,5], and employ the modified Stoner criterion. We have also found that strong-coupling theories (which map onto effective Falicov-Kimball models) display pathological behavior in the magnetic transition temperature, because the (approximate) irreducible vertex functions do not reproduce the atomic limit when $U / t^{*} \rightarrow \infty$. Finally, we have shown that much of the behavior found in $\mathrm{Cr}$ and dilute $\mathrm{Cr}$ alloys can be described by an effective single-band Hubbard model that does not include any of the microscopic details of the band structure.

We would like to acknowledge useful discussions with B. Goodman, L. Falicov, V. Janiš, Th. Pruschke, R. Scalettar, D. Scalapino, P. van Dongen, D. Vollhardt, and J. Wilkins. This work was supported by the National Science Foundation Grants No. DMR-9107563 and No. PHY-8904035 and by the Office of Naval Research Grant No. N00014-93-1-0495. In addition M. J. would like to acknowledge the support of the NSF NYI program. Computer support was provided by the Ohio Supercomputer Center.

*Present address: Department of Physics, Georgetown University, Washington, DC 20057-0995.

[1] E. Fawcett, Rev. Mod. Phys. 60, 209 (1988); E. Fawcett et. al., Rev. Mod. Phys. 66, 25 (1994), and references therein.

[2] J. Hubbard, Proc. R. Soc. London A 276, 238 (1963).

[3] W.C. Koehler et. al., Phys. Rev. 151, 405 (1966); A. L. Trego and A. R. Mackintosh, Phys. Rev. 166, 495 (1968).

[4] D. R. Penn, Phys. Rev. 142, 350 (1966).

[5] H. J. Schulz, Phys. Rev. Lett. 64, 1445 (1990).

[6] W. Metzner and D. Vollhardt, Phys. Rev. Lett. 62, 324 (1989).

[7] J.E. Hirsch and R.M. Fye, Phys. Rev. Lett. 56, 2521 (1986).

[8] E. Müller-Hartmann, Z. Phys. B 74, 507 (1989); 76, 211 (1989).

[9] M. Jarrell, Phys. Rev. Lett. 69, 168 (1992).

[10] P. G. J. van Dongen, Phys. Rev. Lett. 67, 757 (1991); A. Martín-Rodero and F. Flores, Phys. Rev. B 45, 13008 (1992).

[11] J. K. Freericks, Phys. Rev. B 50, 403 (1994).

[12] V. Janiš and D. Vollhardt, Int. J. Mod. Phys. B 6, 731 (1992); Z. Phys. B 91, 317 (1993); V. Janiš, J. Mašek, and D. Vollhardt, Z. Phys. B 91, 325 (1993).

[13] Y. M. Li and N. d'Ambrumenil, Mod. Phys. Lett. B 6, 1827 (1992); Phys. Rev. B 49, 6058 (1994).

[14] L. M. Falicov and J. C. Kimball, Phys. Rev. Lett. 22, 997 (1969).

[15] J. K. Freericks and L. M. Falicov, Phys. Rev. B 41, 2163 (1990); P. Lemberger, J. Phys. A 25, 715 (1992); J. K. Freericks, Phys. Rev. B 47, 9263 (1993).

[16] P. G. J. van Dongen (unpublished). 